\title{
Becoming Art. Exploring Cross-Cultural Categories de Howard MORPHY
}

\section{Raymond MAYER}

\section{(2) OpenEdition}

\section{Journals}

\section{Édition électronique}

URL : http://journals.openedition.org/jso/5980

DOI : 10.4000/jso.5980

ISSN : $1760-7256$

\section{Éditeur}

Société des océanistes

\section{Édition imprimée}

Date de publication : 15 décembre 2009

Pagination : 337-338

ISBN : 978-2-85430-026-0

ISSN : 0300-953x

\section{Référence électronique}

Raymond MAYER, "Becoming Art. Exploring Cross-Cultural Categories de Howard MorPHY », Journal de la Société des Océanistes [En ligne], 129 | juillet-décembre 2009, mis en ligne le 15 décembre 2009, consulté le 24 septembre 2020. URL : http://journals.openedition.org/jso/5980 ; DOI : https://doi.org/ $10.4000 / j s 0.5980$ 
ne mérite d'être connu qu'à titre de contre-exemple et de repoussoir.

Gilles BounOuRE

Morphy Howard, 2007. Becoming Art. Exploring Cross-Cultural Categories, Oxford \& New-York, Berg Publishers, 234 p., bibliogr., index, notes, 59 fig. en noir et blanc.

Howard Morphy, directeur de l'École de recherche en humanités à l'Université nationale australienne de Canberra (ANU), tire parti de son expérience de terrain pour remettre en question l'ensemble des catégories d'analyse appliquées à la pratique artistique en contexte ethnique. Son expérience spécifique du Nord de l'Australie, en Terre d'Arnhem, et plus particulièrement auprès des Yolngu, n'est pas le résultat de la rencontre d'un jour, mais d'une longue fréquentation de trente-cinq ans (1973-2008). Mieux, il a été le témoin du changement radical d'appréciation occidentale de la production artistique locale, d'abord considérée comme simple artisanat ethnique (à la limite de l'artefact d'aéroport pour touristes) avant d'être reconnue comme art justiciable du qualificatif de « beaux-arts » ( fine art », p. XI) et comme tel classable dans les grandes collections de niveau mondial. De là, la limpidité du titre de l'ouvrage Becoming Art dédié à ce renversement de perspective (comment ce qui n'était pas vu comme art est, à la génération actuelle, " en train de devenir art»), et du sous-titre stipulant une «exploration de catégories transculturelles ». Autrement dit, l'auteur ne se contente pas d'un compte rendu de terrain, il ambitionne d'en dégager une nouvelle théorie. L'ouvrage analyse le passage du local au global, tout en évitant le piège de la réduction du «global » à l'ethnocentrisme occidental élevé au rang d'un universalisme de jure.

La mise en cause de l'ethnocentrisme de la critique d'art n'est évidemment pas nouvelle. Il y a plus d'un demi-siècle, Claude Lévi-Strauss, par exemple, tenait des propos comparables sur les productions « ethniques » de la Colombie britannique et proférait ce jugement alors anticipateur :

« L'époque n'est pas lointaine, sans doute, où les collections provenant de cette partie du monde quitteront les musées ethnographiques pour prendre place dans les musées des beaux-arts, entre l'Égypte ou la Perse antiques et le Moyen Âge européen. » (1943 : 176)

Une véritable profession de foi, avant la lettre, pour l'intégration des " arts premiers » dans les collections du Louvre, voire un exercice divinatoire pour susciter la création d'un musée du quai Branly privilégiant la dimension esthétique d'objets autrefois simplement ethnographiques!

Ce n'est donc pas tout à fait un hasard si la première figure - sur les cinquante-neuf que recèle l'ouvrage d'Howard Morphy - nous offre justement un coin de plafonds et murs du musée du quai Branly aux couleurs aborigènes, et sur fond de Tour Eiffel en cadrage oblique - cela ne s'invente pas ! L'inspiratrice austra- lienne de ces peintures murales, $\mathbf{M}^{\mathrm{me}}$ Gulumbu Yunupingu, de culture yolngu, apparaît elle-même au premier plan de cette figure, nous permettant de mettre un nom et un visage sur l'œuvre réalisée, ce qui est précisément le propre de toutes les œuvres cotées sur le marché de l'art. Je reviendrai sur l'iconographie de l'ouvrage un peu plus loin. Ce qu'il y a de convaincant dans la démarche de Howard Morphy, c'est que celle-ci va jusqu'au bout de son propos et qu'elle y va même doublement, à la fois en explorant toutes les facettes possibles des catégorisations applicables aux œuvres d'art, et en parcourant jusqu'à son terme l'expérience singulière de la production yolngu de l'Australie du Nord. Pratiques et théories sont dévoilées et analysées d'un même regard.

Les trois premiers chapitres (pp. 27-86) nous installent dans l'histoire de l'art spécifique des Yolngu, de sa période la plus ancienne qui est rapportée par l'auteur au XVIII ${ }^{\mathrm{e}}$ siècle, jusqu'à sa prise en considération dans la catégorie des «beaux-arts» («fine arts») qui se situe au milieu du $\mathrm{Xx}^{\mathrm{e}}$ siècle. Cette première partie est essentiellement descriptive. L'on y apprend que les premières collections d'objets furent constituées par des missionnaires (révérend Chaseling en 1937, p. 48) puis par des anthropologues et des scientifiques (Berndt en 1946, Mountford en 1948, p. 51), avant d'être promues par une exposition spécialement dédiée à l'art aborigène (galerie d'art David Jones à Sydney en 1949, p. 53). Mais le tournant de cette histoire d'art est à dater, selon Morphy, de 1958, année de la décision d'un chirurgien, Stuart Scougall, et d'un directeuradjoint de musée, Tony Tuckson, d'admettre des collections aborigènes à l'Art Gallery of New South Wales (p. 54). Décision historique qui, cette fois-là, a précédé de quelque quarante ans l'initiative du Louvre ou du quai Branly, puisqu'en faisant entrer les œuvres au musée des beaux-arts en lieu et place du musée ethnographique, on s'est mis à requalifier des œuvres « folkloriques » en œuvres d'art à part entière. Pour Morphy, même s'il ne partage pas la distinction sèche entre « artistique » et « ethnographique » (p. 55), le changement d'affectation spatiale fut le signal traduisant un changement de catégorisation dans le classement des œuvres. Cette question est reprise en détail jusqu'à son chapitre 8 , mais elle est transposée dans l'intervalle sur un plan théorique.

La deuxième partie du livre (pp. 87-172) ouvre ainsi la discussion théorique sur les questions de représentation (chap. 5), de style et de signification (chap. 6), ainsi que de discours de légitimation en matière artistique (chap. 7). Sur le plan théorique, la nouveauté de perspective réside dans le positionnement de l'artiste au centre de la fixation de la valeur, et non plus à la périphérie du marché de l'art. Selon l'auteur, le critique d'art n'a plus le monopole du jugement artistique, mais l'artiste local entre dans la dynamique du changement de catégorisation et joue un rôle actif dans la création artistique répondant aux critères internationaux.

Une troisième partie de l'ouvrage, bien que plus succincte (pp. 173-195), peut alors librement s'adonner à appliquer les conclusions tirées de la discussion théorique à la situation de l'artiste local face aux 
égaleries d'art mondiales. Le chapitre de conclusion proprement dit (pp. 187-195) ne se prive pas de capitaliser les acquis de la démonstration faite sur l'exemple australien des Yolngu pour l'étendre à des cas de figure qui transgressent allègrement toutes sortes de frontières, d'ailleurs souvent plus idéologiques et financières que culturelles et historiques.

Partant d'une analyse processuelle d'un exemple concret, il aboutit à une claire détermination de la genèse de la catégorie d' " art », non seulement comme catégorie exogène, mais aussi comme catégorie endogène. Car l'innovation corollaire, c'est l'alternative positive qu'il propose, sous la forme de la possibilité de transformation, à la fois endogène et exogène, d'une conception esthétique dont le blocage transculturel initial est levé. Nul ne doute qu'il s'agisse d'une question importante, puisque concernant potentiellement dix mille cultures, et pas seulement les cultures australiennes directement analysées.

Questionnant les catégories d'analyse généralement produites pour appréhender l'art aborigène, l'auteur revendique le double angle critique d'une approche anthropologique et d'une histoire de l'art pour discuter la pertinence du discours occidental sur des œuvres non occidentales. Il ne fait pas que dénoncer un perpétuel ethnocentrisme, mais il offre une alternative fondée sur un argumentaire méthodiquement établi. Si l'on suit le cheminement retracé par Howard Morphy sur un exemple australien, force est de conclure que c'est une décision individuelle de directeur de musée de beaux-arts qui a été déterminante dans la réévaluation de la production artistique précédemment considérée comme simplement « ethnique ». Si on voulait le dire plus simplement, on dira qu'on est passé d'un art " populaire » ou « folklorique », le plus souvent anonyme, à un art « élitiste », individualisé et coté sur le marché international de l'art. La démonstration théorique de Howard Morphy montre deux choses : que le concept d'art est historiquement situable, quelles que soient les cultures en présence; que les producteurs d'art sont associés ou associables à l'inscription d'une production artistique à un niveau mondial. Sur ce point, Howard Morphy propose une définition transculturelle de l'art incluant dimension esthétique et dimension sémantique (p. XI et passim). Même si certains auteurs contestent toute catégorisation occidentale ségrégative de l'art et y dénoncent son " hyper-sémantisation » (par exemple, Laplantine, 2009), on doit reconnaître que l'analyse de Morphy reste un des exemples les plus aboutis d'une observation fine associée à un effort de théorisation transculturelle.

Revenons cependant à l'iconographie du livre qui contient cinquante-neuf figures présentées exclusivement en noir et blanc, et partagées entre des photographies d'événements de terrain et des photographies reproduisant des œuvres d'artistes. Si la première série des illustrations ne souffre pas de leur édition en noir et blanc, la seconde série, celle des œuvres, oblige normalement à recourir à une édition en couleur. On ne comprendrait pas que l'on puisse par exemple présenter uniquement par des illustrations en noir et blanc la peinture italienne de Canaletto ou d'un artiste contemporain coté. Il est aujourd'hui relativement facile d'incorporer un cahier de planches en couleur dans n'importe quel ouvrage. Ne pas le faire, dans le cas particulier de cette étude d'art australien, pourrait signifier que le chromatisme n'est pas pertinent dans les peintures " aborigènes ", ou que la lecture de la couleur est réduite au contraste entre une couleur plus claire et une couleur plus foncée (ce que donne effectivement le rendu d'une illustration en noir et blanc), mais ce qui est évidemment un contre-sens par rapport à ce que Howard Morphy veut nous donner à comprendre de l'art australien.

De plus, l'art coté impose ses règles de présentation des œuvres : outre le nom du créateur, le titre et la date de l'œuvre, il est d'usage d'en indiquer les matériaux utilisés et les dimensions. Ici on peine à savoir ce qui est tapisserie, écorce peinte ou peinture murale. Il est à mon avis contre-productif de vouloir démontrer le rehaussement de l'art ethnique à un niveau d'art coté, en ne respectant pas le code de présentation des œuvres cotées. Revenir au noir et blanc " ethnographique », alors que les « beaux-arts » travaillent expressément en couleur est, me semble-t-il, la seule « erreur » matérielle de ce livre. L'édition, dans sa version actuelle, dessert le dessein théorique.

Au-delà des points d'articulation de l'argumentaire, ce qui retient l'attention c'est la perspective globale qui se dégage de cette pénétrante étude locale. La proposition principale consiste à placer les artistes euxmêmes au centre de l'argumentaire critique. Ce nouveau recentrement est à la clé du nouveau déploiement de la critique de l'œuvre d'art (ou jugée comme telle). La question du clivage entre art populaire et art de cotation n'est sans doute pas épuisée, et cette étude n'est ni la première - celle-ci étant attribuée à l'historien de l'art Aby Warburg considérant dès 1895 que la culture artistique hopi de l'Arizona est « aussi élaborée que la peinture italienne de la Renaissance " (réédition 2003, citée par Laplantine, 2009) -, ni la dernière à s'y attacher; mais elle constitue indéniablement une contribution théorique majeure à la catégorisation des œuvres d'art ethnique et post-ethnique dans un monde globalisé.

\section{RÉFÉRENCES CITÉES}

LÉVI-STRAuss Claude, 1943. The Art of the Northwest Coast at the American Museum of Natural History, Gazette des Beaux-Arts, New-York, pp. 175-182.

LAPlantine François, 2009. Son, images et langage. Anthropologie esthétique et subversion, Paris, Beauchesne.

Warburg Aby, 2003. Le rituel du serpent. Art et anthropologie, Paris, Macula.

Raymond MAYER, Université Omar Bongo de Libreville et Université de Lyon 2 